\title{
Sugammadex: An Update
}

\author{
Tiberiu Ezri1,2*, Mona Boaz ${ }^{3}$, Alexander Sherman ${ }^{1}$, Marwan Armaly4, Yitzhak Berlovitz ${ }^{5}$ \\ 1 Departments of Anesthesia, Wolfson Medical Center, affiliated to Tel Aviv University, Tel Aviv, Israel \\ 2 Outcomes Research Consortium, Cleveland, OH, USA, \\ 3 Faculty of Nutrition Sciences, Ariel University, Ariel, Israel \\ ${ }^{4}$ Department of Anesthesiology, the Baruch Padeh Medical Center, Poriya, affiliated to the Faculty of Medicine in the \\ Galilee- Bar Ilan University, Israel \\ 5 Department of Management, Wolfson Medical Center, affiliated to Tel Aviv University, Tel Aviv, Israel
}

\section{ABSTRACT}

The purpose of this update is to provide recent knowledge and debates regarding the use of sugammadex in the fields of anesthesia and critical care. The review is not intended to provide a comprehensive description of sugammadex and its clinical use.

Keywords: muscle relaxants, aminosteroidal group, reversal, neostigmine, sugammadex

Received: 18 December 2015 / Accepted: 10 January 2016

\section{INTRODUCTION}

Sugammadex is used to reverse neuromuscular blockade produced by the aminosteroid neuromuscular blocking drugs rocuronium, vecuronium and pancuronium [1] through encapsulation and inactivation of these muscle relaxants. It is a gamma-cyclodextrin, consisting of oligosaccharides linked around a central cavity. The muscle relaxant becomes entrapped within this cavity within a short time after sugammadex administration, neutralizing the relaxants, decreasing their plasma level and creating a concentration gradient between the neuromuscular end plate and plasma. This gradient causes displacement of the muscle relaxant from the end plate back into the plasma and further neutralization of the remaining relaxant. This mechanism of action explains the rapid reversal effect of sugammadex. In contrast to neostigmine, sugammadex can, through this unique mechanism, reverse even deep muscle relaxation, in a dose-dependent manner.

\section{WHY PREFER SUGAMMADEX?}

Sugammadex is devoid of the muscarinic side-effects of neostigmine. Its reversal effect is more predictable and, as previously stated, can reverse even deep blocks.
Residual paralysis (curarization) may increase postoperative respiratory morbidity by impairing coughing, swallowing and the patients' ability to breathe deeply. Residual paralysis occurs more frequently with neostigmine than with sugammadex.

In a recent prospective, multicenter study [2] of the incidence and severity of residual paralysis following surgery, residual curarization (a TOF ratio< $<0 \%$ ) was detected by the accelerographic method (TOFWatch $^{\circledast}$ ), immediately before extubation and upon patient's arrival at the Post Anesthesia Care Unit (PACU). In this study, rocuronium was used in $99 \%$ of patients and neostigmine was used for reversal in $74 \%$ of patients, with the remaining patients not receiving any reversal agent. The incidence of residual paralysis was $63.5 \%$ at the point of tracheal extubation and $56.5 \%$ at the point of arrival at the PACU. The authors concluded that residual paralysis is common at the time of tracheal extubation and arrival at the PACU, despite using qualitative neuromuscular monitoring and neostigmine. It was concluded that more effective detection and management of neuromuscular block is needed to reduce the risks associated with residual curarization.

What do we know about the incidence of residual paralysis after sugammadex reversal? In a recently pub-

\footnotetext{
Correspondence to: Tiberiu Ezri, Departments of Anesthesia, Wolfson Medical Center, affiliated to Tel Aviv University, Tel Aviv, Israel. Holon, 62 Ha-Lokhamim, 58100 , Israel. E-mail: tezri@bezeqint.net

Mona Boaz, Department of Nutrition Sciences, School of Health Sciences, Ariel University, Ariel, Israel. E-mail: mboaz8@yahoo.com

Alexander Sherman, Departments of Anesthesia, Wolfson Medical Center, Holon, 62 Ha-Lokhamim, 58100, affiliated to Tel Aviv University, Israel. Email: shuranet@012.net.il

Marwan Armaly, Department of Anesthesiology, the Baruch Padeh Medical Center, Poriya, affiliated to the Faculty of Medicine in the Galilee - Bar Ilan University, Israel. The Baruch Padeh

Medical Center, Poriya, M.P, The lower Galilee 15208. Email: tlevkovich@poria.health.gov.il

Yitzhak Berlovitz, Department of Management, Wolfson Medical Center, Holon, 62 Ha-Lokhamim, 58100, affiliated to Tel Aviv University, Israel. Email: berlo@wolfson.health.gov.il
} 
lished randomized study of 150 patients undergoing abdominal surgery [3] sugammadex was compared to neostigmine in terms of residual paralysis incidence in the PACU. No patient had residual paralysis after reversal with sugammadex ( 0 out of 74 ) compared to $43 \%$ after neostigmine (33 out of 76) usual care patients. However, it should be emphasized that residual paralysis may still occur after reversal with sugammadex if a lower than recommended doseis administered [4].

\section{- INCREASING WORLDWIDE USE OF SUGAM-}

\section{MADEX}

Due to the above mentioned advantages over the "historical" anticholinesterase type antagonists, there is a reported increase in the use of sugammadex worldwide. A recent report from Perth, Australia [4], revealed that, sugammadex was introduced in 2011 and is currently used without access restriction. In 2013, 7000 doses of sugammadexwere given, representing a near complete shift to the rocuronium-sugammadex relaxant-antagonist combination. At Wolfson Medical Center in Israel, approximately $20 \%$ of cases are reversed with sugammadex. Of course, in many hospitals, the cost of sugammadex is an important limiting factor when contemplating the comprehensive replacement of neostigmine.

The same report from Australia [4] noted that the "one size fits all" dose of sugammadex may occasionally result in residual paralysis attributed to underdosing. Therefore, further staff education is necessary in this regard.

In December 2015, sugammadex was approved by the FDA (FDA News Release - 15 December, 2015).

\section{AdVerse efFects, Contraindications \& PRECAUtions}

Research regarding adverse effects, contraindications and precautions associated with sugammadex use is ongoing [5].

Among reported adverse effects, the most frequently encountered are bucking \& movement in $3.0 \%$ of patients, dysgeusia (metal or bitter taste) \& parosmia in $1 \%$, bronchospasm in $2.6 \%$, recurrent neuromuscular blockade related to suboptimal dose of sugammadex in $1.3 \%$, and rarely allergic reactions [5]. The recent FDA report (FDA News Release - 15 December, 2015) also mentions as side-effects nausea/ vomiting, hypotension, pain, headache, temporarily reduction of the steroid contraceptives' effect and severe bradycardia responsive to atropine treatment.

There is not enough published data regarding the use of sugammadex in pregnant patients and in children younger than two years, although a few studies have shown successful off-label use of sugammadex in younger patients [6-8]. No dose adjustment of the drug is necessary for elderly patients [5].

\section{TORSADOGENICITY OF SUGAMMADEX}

Reversal of neuromuscular block with anticholinesterase-anticholinergic combinations has been associated with significant QTc prolongation, while such an effect has not been demonstrated for sugammadex, even at high doses [9].

\section{GYPERSENSITIVITY ASSOCIATED WITH SUGAMMADEX ADMINISTRATION}

In a systematic review, including unpublished reports, Tsur and Kalansky [10] identified 15 cases of sugammadex hypersensitivity. Seventy three percent met World Anaphylaxis Organization criteria for anaphylaxis.

All cases occurred within 4-5 minutes subsequent to administration and it was concluded that awareness is required for the possibility of drug-induced hypersensitivity during the critical 5-minutes following sugammadex administration. The true incidence of sugammadex-induced hypersensitivity is not yet determined, though in view of the drug's extensive use, it appears to be a very rare event.

\section{- SUgAMMADEX IN THE MANAGEMENT OF ROCURONIUM-INDUCED ANAPHYLAXIS}

McDonnell [11] described a 33-yr-old female who suffered severe anaphylactic shock after rocuronium administration. After 19 minutes of ineffective traditional management, $500 \mathrm{mg}$ sugammadex was administered with immediate haemodynamic improvement. The mechanism of this beneficial effect is unknown, however, in view of its own potential for causing anaphylaxis, a risk benefit ratio should be considered before treating rocuronium-induced anaphylaxis with sugammadex [12]. 
USE OF SUGAMMADEX IN PATIENTS WITH SEVERE RENAL AND/OR HEPATIC IMPAIRMENT

Severe renal impairment $(\mathrm{CrCl}<30 \mathrm{~mL} / \mathrm{min})$ is a contraindication to sugammadex administration. The same is the case in patients with severe hepatic impairment, especially among patients with coagulopathy, though no studies in patients with severe hepatic failure have been reported [5]. Sugammadex, $4 \mathrm{mg} / \mathrm{kg}$ provided rapid reversal of deep rocuronium-induced block in renal and control patients. However, in renal failure patients, sugammadex-encapsulated rocuronium complex is detectable in plasma seven days after administration. High-flux haemodialysis is effective in removing the sugammadex-encapsulated rocuronium complexes [13].

\section{SUgAMMADEX AND SURGICAL BLEEDING}

While sugammadex produced limited, transient $(<1$ hour) increases in activated partial thromboplastin time and prothrombin time, it was not associated with increased risk of bleeding compared to traditional care modalities [14].

\section{IINDICATIONS FOR THE USE OF SUGAMMA- DEX}

There are numerous clinical conditions where sugammadex reversal is reportedly preferred to reversal with an atropine-neostigmine combination. The list of indications is large and continually growing, as shown by published reports. The following is a list of clinical conditions in which sugammadex is preferentially used as reversal agent at Wolfson Medical Center:

- All bariatric surgery cases and morbidly obese patients undergoing other surgeries

- Unstable angina, tight aortic and tight mitral stenosis

- Residual paralysis after neostigmine reversal

- Myasthenia gravis and muscular dystrophies

- Patients with wasted muscle mass

- Pneumonectomies or other cases with severely limited lung reserves

- Failed intubation with difficult or failed ventilation after the administration of rocuronium, as we no longer use vecuronium and pancuronium
Other possible indications $[15,16]$ are:

- operations terminated prematurely

- surgeries that require profound motor block for a short time (i.e. micro laryngeal surgery)

- other cases that require deep relaxation (i.e. laparoscopy) or whenever other reversal agents are contraindicated or ineffective.

The successful reversal of prolonged motor block after $15 \mathrm{mg}$ total dose of rocuronium for caesarean delivery in a $70 \mathrm{~kg}$ patient treated with magnesium was first reported in 2012 [17]. At the end of surgery, $35 \mathrm{~min}$ utes after rocuronium administration, the patient had a TOF count and a PTC count of 0 . Full motor recovery was re-established 60 seconds after the administration of $5.7 \mathrm{mg} / \mathrm{kg}$ sugammadex [17].

\section{UUSE OF ROCURONIUM AND SUGAMMA- DEX IN MORBIDLY OBESE PATIENTS}

Small case series have shown that rocuronium for induction of anesthesia and sugammadex for reversal may be safe and effective in pregnant $[18,19]$ and morbidly obese patients [20].

The elimination of rocuronium in morbidly obese patients may be delayed in those with fatty or fibrotic liver and reduced hepatic or renal blood flow.

There is some controversy about dosing of sugammadex in morbidly obese patients. Most authors recommend rocuronium dosing by ideal body weight and sugammadex dosing by IBW $+40 \%$ [21].

Following neostigmine reversal, the time from the appearance of $\mathrm{T}_{2}$ in TOF count to TOFR 0.9 is much longer, 26 minutes in obese patients compared to 7 minutes in nonobese patients [22] and the "unsafe period of recovery", given as the time from visual loss of fade to TOF 0.9 , is 0.3 minutes with sugammadex compared to 10 minutes with neostigmine [23]. Overall, sugammadex appears to be a safer and more predictable reversal agent in obese patients.

So far, the effect of reversal with sugammadex compared to neostigmine on postoperative respiratory complications has not been fully elucidated. In a retrospective study of 179 patients who had undergone laparoscopic sleeve gastrectomy, Ezri at al [24] have found no difference between the two groups in regard to the incidence of respiratory complications. Large, prospective, randomized trials are necessary to find the true advantage of sugammadex over neostigmine reversal 
in decreasing the incidence of postoperative respiratory complications.

\section{RAPID SEQUENCE INDUCTION (RSI) WITH ROCURONIUM}

While some authors consider that RSI with rocuronium is associated with fewer complications and better oxygenation during apnea, others report that succinylcholine provides better intubation conditions and shorter recovery time of failed intubation-failed ventilation scenarios [25-27]. These issues remain controversial and await further evidence.

In a randomized prospective study of 60 patients, Sørensen et al. [28] observed how rapidly spontaneous ventilation was re-established after RSI with either succinylcholine $(1 \mathrm{mg} / \mathrm{kg})$ or rocuronium $(1 \mathrm{mg} /$ $\mathrm{kg}$ ) followed by $16 \mathrm{mg} / \mathrm{kg}$ sugammadex. Spontaneous ventilation returned 406 seconds after succinylcholine compared to 216 seconds after rocuronium-sugammadex. The time to T1 $90 \%$ was 518 seconds with succinylcholine and 168 seconds with rocuroniumsugammadex. Conditions and time to intubation were not different between the two groups. The authors concluded that RSI with rocuronium-sugammadex combination allowed earlier spontaneous ventilation than with succinylcholine.

\section{ROCURONIUM INDUCTION AND DIFFICULT}

\section{AIRWAY}

Sørensen [28] has suggested that RSI with rocuronium is safe if sugammadex is readily available in case of failed airway management, even in patients with a difficult airway.

The induction with rocuronium in patients with predicted difficult airway may be too risky and sugammadex rescue reversal is recommended to be retained for use in unanticipated difficult airways [29]. When facing a failed intubation - failed ventilation scenario following rocuronium induction, practitioners need to have an appropriate dose of sugammadex immediately available. A "wake-up" decision has to be taken within a few minutes [29,30].

However, sugammadex administration does not guarantee return of spontaneous ventilation or easy mask ventilation and effective oxygenation, despite regaining motor strength. Other factors that may prevent effective oxygenation during recovery are airway oedema from repeated intubation attempts and mechanical airway closure due to laryngospasm or the presence of a foreign body.

The inappropriateness of using rocuronium in the case of a predicted difficult airway is demonstrated by several case reports where sugammadex was not helpful in obtaining effective oxygenation following failed intubation $[31,32]$.

Therefore, we believe that whenever a difficult airway is suspected, "awake" fiber optic intubation may be a safer alternative to induction with rocuronium.

\section{USE OF SUGAMMADEX IN THE ICU AND TRAUMA PATIENTS}

Gradually, sugammadex has been gaining popularity in intensive care (ICU) settings [33]. In ICU patients, sugammadex may be used in patients for whom succinylcholine is contraindicated, such as prolonged bedridden patients with disuse atrophy, burns, or major trauma. Other indications for sugammadex in the ICU include reversal of neuromuscular blockade given for short procedures where muscle relaxants are required or the treatment of residual neuromuscular blockade. The use of rocuronium for RSI in trauma patient, followed if necessary by sugammadex has been suggested by Ortega-Gonzalez [34].

\section{- CONCLUSIONS}

Sugammadex is gaining popularity in the fields of anesthesia and intensive care. Devoid of the side effects of neostigmine and due to its ability to rapidly reverse even deep muscle relaxation, sugammadex seems to be superior to neostigmine as a reversal agent. Thus, it has the ability to save patients' lives following failed intubation - failed ventilation after rocuronium induction. However, elective rocuronium induction in patients with predicted difficult intubation is not recommended since sugammadex is not an absolute guarantee for a safe outcome in all failed intubation - failed ventilation scenarios.

\section{DECLARATION OF CONFLICT OF INTEREST}

Prof. Ezri has been invited speaker at scientific meetings in Israel, sponsored by MSD Pharmaceutical Company. 


\section{REFERENCES}

1. Shields M, Giovannelli M, Mirakhur K, et al. Org 25969 (sugammadex), a selective relaxant binding agent for antagonism of prolongedrocuronium-induced neuromuscular block. Br J Anaesth. 2006;96:36-43.

2. Fortier LP, McKeen D, Turner K, de Medicis E, et al. The RECITE Study: A Canadian Prospective, Multicenter Study of the Incidence and Severity of Residual Neuromuscular Blockade. AnesthAnalg. 2015;121:366-72.

3. Brueckmann B, Sasaki N, Grobara P, et al. Effects of sugammadex on incidence of postoperative residual neuromuscular blockade: a randomized, controlled study. Br J Anaesth. 2015;115:743-51.

4. Ledowski T, Ong JS, FlettT. Neuromuscular Monitoring, Muscle Relaxant Use, and Reversal at a Tertiary Teaching Hospital 2.5 Years after Introduction of Sugammadex: Changes in Opinions and Clinical Practice. Anesthesiol Res Pract. 2015;2015:367937.

5. Partownavid P, Romito BT, Ching W, Berry AA, Barkulis CT, Nguyen KP, JahrJS. Sugammadex: A Comprehensive Review of the Published Human Science, Including Renal Studies. Am J Ther.2015;22:298-317.

6. Benigni A, Maffioletti M, Spotti A, Benigni AM, Locatelli BG, Sonzogni V. Efficacy and safety of a sugammadex dose of $4 \mathrm{mg} /$ $\mathrm{kg}$ in early reversal of a deep neuromuscular block rocuroniuminduced in infants and children: a case series. Eur J Anaesthesiol. 2013;30:161-2.

7. Plaud B, Meretoja O, Hofmockel R, et al. Reversal of rocuroniuminduced neuromuscular blockade with sugammadex in pediatric and adult surgical patients. Anesthesiology. 2009;110:284-94.

8. Meretoja OA. Neuromuscular block and current treatment strategies for its reversal in children. Paediatr Anaesth. 2010;20:591-604.

9. Staikou C, Stamelos M, Stavroulakis E. Impact of anaesthetic drugs and adjuvants on ECG markers of torsadogenicity. $\mathrm{Br} \mathrm{J}$ Anaesth. 2014;112:217-30.

10 Tsur A, Kalansky A. Hypersensitivity associated with sugammadex administration: a systematic review.Anaesthesia. 2014;69:1251-7.

11. McDonnell NJ, Pavy TJ, Green LK, Platt PR. Sugammadex in the management of rocuronium-induced anaphylaxis. Br J Anaesth. 2011;106:199-201.

12. Plaud B. A new option for the treatment of anaphylaxis linked to steroidal neuromuscular blockers: How much value should we grant to case reports? Can J Anaesth. 2014;61:511-8.

13. Cammu G, Van Vlem B, van den Heuvel M, et al. Dialysability of sugammadex and its complex with rocuronium in intensive care patients with severe renal impairment. $\mathrm{Br} J$ Anaesth. 2012;109:382-90.

14. Rahe-Meyer N, Fennema H, Schulman S, et al. Effect of reversal of neuromuscular blockade with sugammadex versus usual care on bleeding risk in a randomized study of surgical patients. Anesthesiology.2014;121:969-77.

15. WATAG (Western Australian Therapeutic Advisory Group):
Sugammadex Guidelines - July 2013 (online source) http://www.watag.org.au/watag/docs/SUGAMMADEX_ Guidelines\%20and\%20Audit\%20July13.pdf.

16. Veelo DP, Gisbertz SS, Hannivoort RA, et al. The effect of ondemand vs deep neuromuscular relaxation on rating of surgical and anaesthesiologic conditions in patients undergoing thoracolaparoscopicesophagectomy (DEPTH trial): study protocol for a randomized controlled trial. Trials. 2015;16:331.

17. Evron S, Yakobashvili S, Rigini N, Ezri T. Successful Reversal with Sugammadex of Deep Neuromuscular Block Caused by Rocuronium and Magnesium Sulfate in a Patient with H.E.L.P Syndrome. Acta Medica Marisiensis. 2012;58:7.

18. Pühringer FK, Kristen $P$, Rex C. Sugammadex reversal of rocuronium-induced neuromuscular block in caesarean section patients: a series of seven cases. Br J Anaesth. 2010;105:657-60.

19. Williamson RM, Mallaiah S, Barclay P. Rocuronium and sugammadex for rapid sequence induction of obstetric general anaesthesia. Acta Anaesthesiol Scand. 2011;55:694-9.

20. Gaszynski T, Szewczyk T, Gaszynski W. Randomized comparison of sugammadex and neostigmine for reversal of rocuroniuminduced muscle relaxation in morbidly obese undergoing general anaesthesia. Br J Anaesth. 2012;108:236-9.

21. Van Lancker P, Dillemans B, Bogaert T, Mulier JP, De Kock M, Haspeslagh $\mathrm{M}$. Ideal versus corrected body weight for dosage of sugammadex in morbidly obese patients.Anaesthesia. 2011;66:721-5.

22. Suzuki T, Masaki G, Ogawa S. Neostigmine-induced reversal of vecuronium in normal weight, overweight and obese female patients. Br J Anaesth. 2006;97:160-3.

23. Illman HL, Laurila $\mathrm{P}$, Antila $H$, Meretoja OA, Alahuhta $S$, Olkkola KT. The duration of residual neuromuscular block after administration of neostigmine or sugammadex at two visible twitches during train-of-four monitoring. Anesth Analg. 2011;112:63-8.

24. Ezri T, Evron S, Petrov I, Schachter P, Berlovitz P, Shimonov M. Residual curarization and postoperative respiratory complications following laparoscopic sleeve gastrectomy. The effect of reversal agents: sugammadex vs. neostigmine. J Crit Care Med. 2015;1:61-67.

25. El-Orbany M, Connolly LA. Rapid sequence induction and intubation: current controversy. Anesth Analg. 2010;110:1318-25.

26. Taha SK, El-Khatib MF, Baraka AS, et al. Effect of suxamethonium vs rocuronium on onset of oxygen desaturation during apnoea following rapid sequence induction. Anaesthesia. 2010;65:358-61.

27. Donati F. Neuromuscular blocking agents. In Barash PG, Cullen BF, Stoelting RK, Cahalan MK, Stock MC, Ortega R: Clinical anesthesia, 7th ed., chapter 20, page 533, Wolters Kluwer, Philadelphia 2013.

28. Sorensen M.K, Bretlau C, Gatke MR, Sorensen AM, Rasmussen LS. Rapid sequence induction and intubation with rocuroniumsugammadex compared with succinylcholine: A randomized trial. Br J Anaesth. 2012;108:682-9. 
Available online at: www.jccm.ro

29. Mendonca C. Sugammadex to rescue a 'can't ventilate' scenario in an anticipated difficult intubation: is it the answer? Anaesthesia. 2013;68:795-9.

30. Bisschops MMA, Holleman C, Huitink JM. Can sugammadex save a patient in a simulated 'cannot intubate, cannot ventilate' situation? Anaesthesia. 2010;65:936-41.

31. Curtis R, Lomax S, Patel B. Use of sugammadex in a 'can't intubate, can't ventilate' situation. $\mathrm{Br} J$ Anaesth.
The Journal of Critical Care Medicine 2016;2(1) • 21

2012;108:612-4

32. Kyle BC, Gaylard D, Riley RH. A persistent 'can't intubate, can't oxygenate' crisis despite rocuronium reversal with sugammadex. Anaesth Intensive Care. 2012;40:344-6.

33. Karalapillai D, Kaufman M, Weinberg L. Sugammadex. Crit Care Resusc. 2013;15:57-62.

34. Ortega-Gonzalez M. Anaesthesia for trauma patients. S Afr Fam Pract. 2012;54(Suppl 1):S2-S6. 